\title{
Desmoid Tumor of the Rectus Abdominis Muscle in a 2-Year-Old Boy
}

\author{
Asmir Jonuzi ${ }^{*}$, Zlatan Zvizdić ${ }^{1}$, Nusret Popović1, Emir Milišić ${ }^{1}$, Azra Karamustafić ${ }^{\text {, Amira Mesic }}{ }^{2}$, \\ Irmina Sefić ${ }^{3}$, Amra Džananović ${ }^{3}$ \\ 1 Clinic of Pediatric surgery, Clinical Center University in Sarajevo, Patriotske lige 81, 71000 Sarajevo, Bosnia and Herzegovina \\ 2 Clinic for Anaesthesiology and Reanimation, Clinical Center University in Sarajevo, Bolnička 25, 71000 Sarajevo, Bosnia and Herzegovina \\ 3 Clinic of Radiology, Clinical Center University in Sarajevo, Bolnička 25, 71000 Sarajevo, Bosnia and Herzegovina
}

\begin{abstract}
Aggressive fibromatosis, also known as desmoid tumor, is a locally invasive soft tissue lesion arising from connective tissues. Reports in children less than 10 years of age is rare. We report a case of a desmoid tumor located at the middle and lower third of the left rectus abdominis, in a 2-year-old-boy. Partial resection of the muscle segment and simultaneous reconstruction of the abdominal wall by abdominal fascia was done. The patient had an uneventful recovery. At one year of follow-up neither recurrence nor functional or aesthetic complications were seen.
\end{abstract}

\section{Keywords: Desmoid tumor; Aggressive fibromatosis; Reconstruction}

Correspondence*: Asmir Jonuzi, Clinic of Pediatric surgery, Clinical Center University in Sarajevo, Patriotske lige 81, 71000 Sarajevo, Bosnia and Herzegovina

E-mail: jonuziasmir@hotmail.com

Submitted: 17-03-2018

Conflict of Interest: None
(C) 2018, Jonuzi et al,

Accepted: 07-05-2018

Source of Support: Nil

This is an open-access article distributed under the terms of the Creative Commons Attribution License, which permits unrestricted use, distribution, and reproduction in any medium provided the original work is properly cited.

\section{INTRODUCTION}

Desmoid tumors, also known as desmoid-type fibromatosis or aggressive fibromatosis, are benign mesenchymal neoplasm characterized by aggressive local infiltration of surrounding tissues, with uncertain growth and increased chances of recurrence, despite no metastatic potential.[1] Although first described by MacFarlane in 1832, the term desmoid was given in 1838 by Muller in Berlin, who first used the term "desmos" relating to the Greek word - "similar to tendon". Desmoid tumor is a rare, deep-seated, musculoaponeurotic tumor of intermediate malignancy with an annual incidence of 0.2 to 0.4 per 100,000 population.[2] These are slowly growing tumors and may take years to get a noticeable mass. It is rarely reported in infants. Herein, we report anterior abdominal wall desmoid in an infant.

\section{CASE REPORT}

A 2-year-old boy presented with a 2-month history of progressively increasing paraumbilical mass. There was no history of trauma and the baby was normal otherwise. Parents decided to seek medical attention on account of abdominal discomfort. The pregnancy, birth and neonatal history were uneventful and he completed the National Programme on Immunization schedule for his age. General physical examination was normal. Physical examination revealed a supra and infra umbili- 
cal painless mass on the left abdominal region next to the umbilicus measuring about $4 \times 2 \mathrm{~cm}$ in diameter, spherical in shape with well-defined edges. The mass was not warm, not tender, firm in consistency and was not attached to skin but attached to underlying structures. Rectal examination was essentially normal. He underwent a Computerized tomography (CT) and magnetic resonance imaging (MRI) of abdomen which showed a $43 \times 12 \times 20 \mathrm{~mm}$, ovoid, heterogeneous mass, restricted to the rectus abdominis sheath and located at the middle and the lower third of the rectus abdominis muscle (Figure 1A). Surgery was performed and it was completely removed with resection of middle and lower rectus muscle. The defect was covered with surrounding fascia. A histopathologic study confirmed the diagnosis of a desmoid tumor with clear surgical margins (Figure 1B, 1C, 1D). The patient had an uneventful recovery. After one year of follow-up, no relapse was seen.

Surgery was performed and it was completely removed with resection of middle and lower rectus muscle. The defect was covered with surrounding fascia. A histopathologic study confirmed the diagnosis of a desmoid tumor with clear surgical margins (Figure 1B, 1C, 1D). The patient had an uneventful recovery. After one year of follow-up, no relapse was seen.
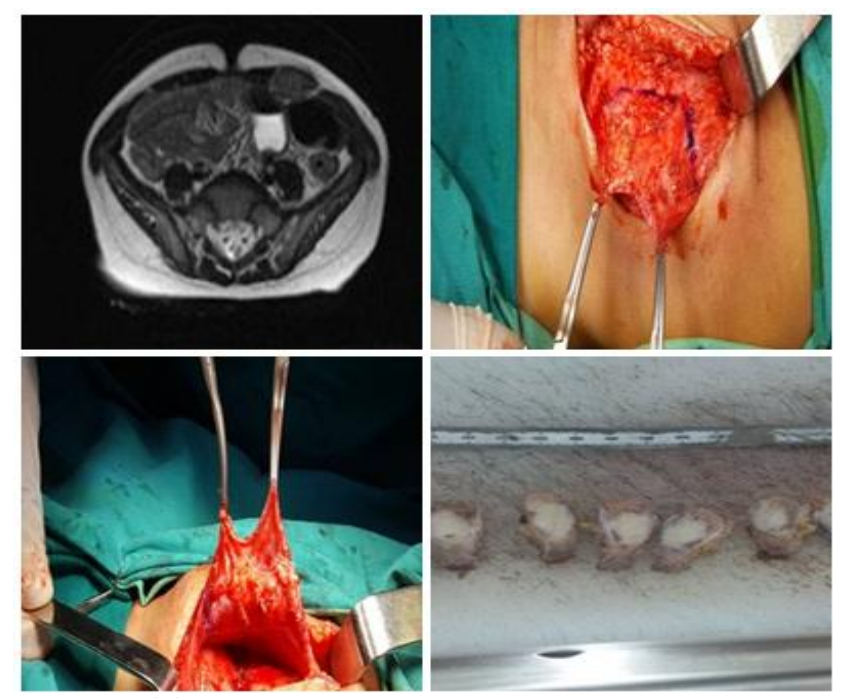

Figure 1: (A): Magnetic resonance showing ovoid lesion and hypodense mass in left rectus abdominis sheath; $(B, C)$ : Perioperative picture; (D): Cut section of the mass.

\section{DISCUSSION}

Desmoid tumors account for $3 \%$ of all soft tissue neoplasms. Desmoid tumors are typically slowly growing mesenchymal tissue; however, they may infiltrate deeper tissue planes thus high chances of recurrence. They often occur in the second or third decade of life but some series have documented desmoid tumors in the middle-aged and elderly.[3]

Occurrence of desmoid tumor in the first decade of life is very rare, our patient was 2-year-old and had noticed the anterior abdominal mass for about 2 month without symptoms and the size had only increased moderately showing the slow-growing nature of the tumor. At surgery, a well-circumscribed tumor mass attached to the inner layer of the rectus abdominis muscle was found and this was excised completely with about $1 \mathrm{~cm}$ tumor margin. In children, a negative margin of resection significantly reduces the recurrence of the tumor [4] however in adults, recurrence can occur in the tumor-free margins.[5]

The diagnosis is based on clinical, radiologic and histopathologic criteria. Its main characteristic is infiltration of the muscles and deep structures. This feature differentiates desmoid tumor from sarcomas.[5] It occurs more commonly in individuals with familial adenomatous polyposis (FAP) occurring as part of an inherited syndrome (Gardner's syndrome).[6] Desmoids arise from myofibroblasts, lacks a true capsule and usually infiltrate into the surrounding muscle. The telomerase length and activity is normal [7], nuclei are small and regular, and mitoses are infrequent - all of which support its histologically benign nature.

Surgery is the mainstay of treatment for desmoid tumors of the abdominal wall. Wide local resection, advocated nearly 100 years ago, remains the treatment of choice for most patients with desmoid tumors. The excision should be completed with $1 \mathrm{~cm}$ margin and the resultant defect is to be repaired with local muscle flaps, distant muscle flaps or by synthetic mesh if necessary.[7,8] Recurrence rates are lower if adequately excised and the morbidity rates associated with the procedure is negligible. Margin-negative resection has historically been the gold standard for treatment of abdominal wall and intra-abdominal desmoid tumors.

To conclude, primary surgery with negative surgical margins was found to be the most successful primary treatment modality for children with a desmoid tumor. A long follow-up is necessary to pick any recurrence.

\section{Consent Statement}

Authors declared that they have taken informed written consent, for publication of this report along with clinical photo- 
graphs/material, from the legal guardian of the patient with an understanding that every effort will be made to conceal the identity of the patient however it cannot be guaranteed.

\section{Author's Contributions}

All authors contributed equally in concept, design, literature review, drafting the manuscript, and approval of the final manuscript.

\section{REFERENCES}

1. Singer S, Maki RG, O'Sullivan B. Soft tissue sarcoma. In: DeVita VT Jr, Lawrence TS, Rosenberg SA: Cancer: Principles and Practice of Oncology. 9th ed. Philadelphia, $\mathrm{Pa}$ : Lippincott Williams \& Wilkins, 2011, pp 1533-77.

2. Reitamo JJ, Scheinin TM, Hayry P. The desmoid syndrome: new aspects in the cause, pathogenesis and treatment of the desmoids tumor. Am J Surg. 1996; 151:230- 7.

3. Stoeckle E, Coindre JM, Longy M, Binh MB, Kantor G, Kind $\mathrm{M}$, et al. Avril A. Bonichon F. Bui BN. A critical analysis of treatment strategies in desmoid tumours: a review of a series of 106 cases. Eur J Surg Oncol. 2009; 35:129-34.
4. Faulkner LB, Hajdu SI, Kher U. Pediatric desmoid tumor: Retrospective analysis of 63 cases. J Clin Oncol. 1995; 13:2813-8.

5. Merchant NB, Lewis JJ, Woodruff JM. Extremity and trunk desmoid tumors: A multifactorial analysis of outcome. Cancer. 1999; 86: 2045-2052.

6. Bonvalot S, Desai A, Coppola S, Le Pechoux C, Terᄀrier P, Domont $\mathrm{J}$, et al. The treatment of des-moid tumors: a stepwise clinical approach. Ann Oncol. 2012; 23:158-66.

7. Middleton SB, Pack K, Phillips RK. Telomere length in familial adenomatous polyposis-associated desmoids. Dis Colon Rectum. 2000; 43:1535-9.

8. Kasper B, Baumgarten C, Bonvalot S, et al. Management of sporadic desmoid-type fibromatosis: a European consensus approach based on patients' and professionals' expertise-a sarcoma patients EuroNet and European Organisation for Research and Treatment of Cancer/Soft Tissue and Bone Sarcoma Group initiative. Eur J Cancer. 2015; 51:127-36. 